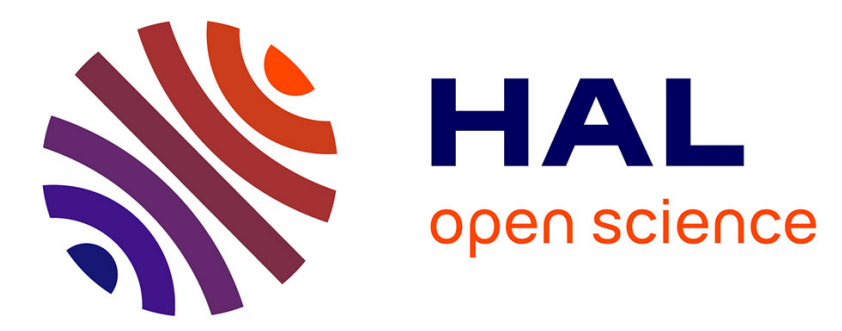

\title{
An LMI-based iterative algorithm for state and output feedback stabilization of discrete-time Lur'e systems
}

\author{
Ariádne L J Bertolin, Pedro L D Peres, Ricardo C L F Oliveira, Giorgio
}

Valmorbida

\section{- To cite this version:}

Ariádne L J Bertolin, Pedro L D Peres, Ricardo C L F Oliveira, Giorgio Valmorbida. An LMIbased iterative algorithm for state and output feedback stabilization of discrete-time Lur'e systems. CDC 2020 - 59th IEEE Conference on Decision and Control, Dec 2020, Jeju Island, South Korea. pp.2561-2566, 10.1109/CDC42340.2020.9303964 . hal-03151882

\section{HAL Id: hal-03151882 \\ https://hal.inria.fr/hal-03151882}

Submitted on 25 Feb 2021

HAL is a multi-disciplinary open access archive for the deposit and dissemination of scientific research documents, whether they are published or not. The documents may come from teaching and research institutions in France or abroad, or from public or private research centers.
L'archive ouverte pluridisciplinaire HAL, est destinée au dépôt et à la diffusion de documents scientifiques de niveau recherche, publiés ou non, émanant des établissements d'enseignement et de recherche français ou étrangers, des laboratoires publics ou privés. 


\title{
An LMI-based iterative algorithm for state and output-feedback stabilization of discrete-time Lur'e systems
}

\author{
Ariádne L. J. Bertolin, Pedro L. D. Peres, Ricardo C. L. F. Oliveira, Giorgio Valmorbida
}

\begin{abstract}
This paper is concerned with the problem of static output-feedback stabilization of discrete-time Lur'e systems. The control law feedbacks both the output and the nonlinearity. By using a quadratic Lyapunov function, new design conditions are provided in terms of new sufficient design linear matrix inequalities where the control gains appear affinely. Using some relaxations, the search for the stabilizing control gains is performed through an iterative algorithm. The approach can be considered as more general than the existing ones thanks to the fact that the gains are treated as decision variables in the optimization problem. Therefore, the approach can handle state or output feedback indistinctly, and can include magnitude or structural constraints (such as decentralization) on the gains. Numerical examples illustrate that the proposed method can provide less conservative results when compared with other techniques from the literature.
\end{abstract}

\section{INTRODUCTION}

The so-called Lur'e system, that is, the feedback connection of a linear time-invariant plant by means of a nonlinear function of the output, subject to sector or slope bounded conditions, can represent a large family of engineering problems [1]. Many researchers have investigated the stability of both continuous- and discrete-time Lur'e systems, in most cases using a quadratic Lyapunov function with an additional term that to take into account the effect of the nonlinearity [2], [3], [4], [5], [6], [7], [8], [9]. In another cases Zames-Falb multipliers is also used as in [10], [11].

Extensions for synthesis conditions are less frequent, even when a standard state feedback control law is investigated. This situation is presented mainly in continuous-time Lur'e systems, that, one possible method is presented in [12], for systems with in sector bounded conditions, that considers saturation and non-linearity feedback (going through saturation or not) in the design structure.

Concerning the design in discrete-time systems, specifically with sector-bounded nonlinearities, there are conditions to obtain the control gains with state and output-feedback controller structure as presented in [13], [14]. Those provides conditions with the presence or not of parametric uncertainties associated Lyapunov method with S-procedure. Another

Supported by the Brazilian agencies: CNPq, CAPES and grants 2019/10947-1, São Paulo Research Foundation (FAPESP).

A. L. J. Bertolin, P. L. D. Peres and R. C. L. F. Oliveira are with the School of Electrical and Computer Engineering, University of Campinas - UNICAMP, 13083-852, Campinas, SP, Brazil. \{ariberto, ricfow, peres\}@dt. fee.unicamp.br.

G. Valmorbida is with the Laboratoire des Signaux et Systèmes, CentraleSupélec, CNRS, University of Paris-Sud, Université Paris-Saclay, Gifsur-Yvette 91192, France. He is also with Inria projet DISCO. (e-mail: giorgio.valmorbidal2s. centralesupelec.fr). approach is based on a nonlinear sampled-data systems as showed in [15], for state and nonlinear-feedback controller in systems with Euler approximate models. The stabilization of swiched systems is also treated as in [16]. Furthermore, in cases with sector and slope restrictions, the robust state feedback controller is proposed in [17].

The conditions are given in terms of linear matrix inequalities (LMIs) [18], providing algorithms with polynomial time and global convergence. However, in most cases, only state feedback control exists and not all the conditions allow the feedback of the nonlinearity.

The aim of this paper is to propose an LMI-based synthesis procedure for output feedback stabilization of discrete-time Lur'e systems. The stabilizing control law is composed by one gain multiplying the measured output and another gain that feedback the output of the nonlinear block. First, a sufficient condition for the existence of stabilizing gains is proposed in terms of LMIs. Besides depending on some matrices that must be given, the condition has as main property the fact that the Lyapunov and the closed-loop matrices (i.e., the control gains) appear affinely in the LMIs. Defining an appropriate choice for the variables that must be given, and relaxations in closed-loop stability and effect of the nonlinearity, a locally convergent iterative algorithm is constructed in terms of the LMI design condition. Examples are provided to compare the performance of the proposed method with the results from other techniques available in the literature.

Notation: For a symmetric matrix, $A>0(A<0)$ means that $A$ is positive (negative) definite. For matrices or vectors $\left({ }^{T}\right)$ indicates the transpose and $\operatorname{He}(A)=A+A^{T}$. The symbol $\star$ represents a symmetric term in a blocked matrix. The identity matrix and the zero matrix are denoted, respectively, by $I$ and 0 . Throughout the text the dimensions of the matrices may be omitted for simplicity (being inferred from the context).

\section{PROBLEM DEFINITION AND PRELIMINARIES}

Consider the discrete-time nonlinear Lur'e system

$$
\begin{aligned}
x(k+1) & =A x(k)+B_{\phi} \phi(z(k))+B_{u} u(k) \\
z(k) & =C_{z} x(k) \\
y(k) & =C_{y} x(k)
\end{aligned}
$$

where $x \in \mathbb{R}^{n_{x}}$ is the state, $u \in \mathbb{R}^{n_{u}}$ is the control input, $y \in \mathbb{R}^{n_{y}}$ is measured output (to be used for feedback) and $z \in \mathbb{R}^{n_{z}}$ is the output that goes through the nonlinearity. Accordingly, 
$A \in \mathbb{R}^{n_{x} \times n_{x}}, B_{\phi} \in \mathbb{R}^{n_{x} \times n_{z}}, B_{u} \in \mathbb{R}^{n_{x} \times n_{u}}, C_{y} \in \mathbb{R}^{n_{y} \times n_{x}}$ and $C_{z} \in$ $\mathbb{R}^{n_{z} \times n_{x}}$.

The time-invariant nonlinearity $\phi: \mathbb{R}^{n_{z}} \rightarrow \mathbb{R}^{n_{z}}$ is decentralized [1] and satisfy the following assumption (sector bounded nonlinearity).

\section{Assumption 1 The nonlinearity $\phi$ verifies}

$$
\phi(z)^{T}[\phi(z)-\Omega z] \leq 0
$$

for all $z \in \mathbb{R}^{n_{z}}$, where $\Omega \in \mathbb{R}^{n_{z} \times n_{z}}$ is a given positive diagonal matrix. Furthermore $\phi(0)=0_{n_{z}}$.

The aim of this paper is to design a stabilizing control law $u$ defined by

$$
u(k)=K y(k)+L \phi(z(k))
$$

where $K \in \mathbb{R}^{n_{u} \times n_{y}}$ is an output-feedback gain and $L \in \mathbb{R}^{n_{u} \times n_{z}}$ is a feedback gain multiplying the nonlinearity, considering Assumption 1, as addressed in next section.

The stabilization conditions used to solve the above problem are formulated in terms of an iterative procedure based on convex optimization, namely a sequence of Semidefinite Programming (SDP). Before presenting the results, Finsler's Lemma [19] is recalled.

Lemma 1 (Finsler's Lemma) Consider matrices $\mathscr{Q} \in \mathbb{R}^{\ell \times \ell}$ and $\mathscr{B} \in \mathbb{R}^{m \times \ell}$, with $\operatorname{rank}(\mathscr{B})<\ell$ and $\mathscr{B} \mathscr{B}_{\perp}=0$. Then, the following statements are equivalent:

i) $\xi^{T} \mathscr{Q} \xi<0, \forall \xi \in \mathbb{R}^{\ell}, \xi \neq 0$, such that $\mathscr{B} \xi=0$;

ii) $\mathscr{B}_{\perp}^{T} \mathscr{Q} \mathscr{B}_{\perp}<0$;

iii) $\exists \mu \in \mathbb{R}$ such that $\mathscr{Q}-\mu \mathscr{B}^{T} \mathscr{B}<0$;

iv) $\exists \mathscr{X} \in \mathbb{R}^{\ell \times m}$ such that $\mathscr{Q}+\mathscr{X} \mathscr{B}+\mathscr{B}^{T} \mathscr{X}^{T}<0$.

\section{MAIN RESUltS}

The following theorem presents a sufficient condition for the design of gains $K$ and $L$ of the control law given in (4) assuring the closed-loop stability of system (1) under Assumption 1 (sector-bounded nonlinearity).

Theorem 1 Let $\bar{Y}_{i} \in \mathbb{R}^{n_{x} \times n_{x}}, i=1,3,4$ and $\bar{Y}_{2} \in \mathbb{R}^{n_{x} \times n_{z}}$, be given matrices. If there exist matrices $K \in \mathbb{R}^{n_{u} \times n_{y}}, L \in$ $\mathbb{R}^{n_{u} \times n_{z}}, Y_{i} \in \mathbb{R}^{n_{x} \times n_{x}}, \quad i=1,3,4, \quad Y_{2} \in \mathbb{R}^{n_{z} \times n_{x}}$ and positive definite matrices $P=P^{T} \in \mathbb{R}^{n_{x} \times n_{x}}$ and $T \in \mathbb{R}^{n_{z} \times n_{z}}$ diagonal, such that

$$
\left[\begin{array}{cccc}
-P & C_{z}^{T} \Omega T & 0 & A_{c l}^{T} \\
\star & -2 T & 0 & B_{c l}^{T} \\
\star & \star & P & -I \\
\star & \star & \star & 0
\end{array}\right]+\operatorname{He}(\underbrace{\left[\begin{array}{c}
Y_{1} \\
Y_{2} \\
Y_{3} \\
Y_{4}
\end{array}\right]}_{=Y} \underbrace{\left[\begin{array}{llll}
\bar{Y}_{1} & \bar{Y}_{2} & \bar{Y}_{3} & \bar{Y}_{4}
\end{array}\right]}_{=\bar{Y}})<0
$$

with

$$
A_{c l}=A+B_{u} K C_{y}, \quad B_{c l}=B_{\phi}+B_{u} L,
$$

then the Lur'e system (1)-(3) in closed-loop is asymptotically globally stable.
Proof: If the inequalities of Theorem 1 hold, then $\operatorname{He}\left(Y_{4} \bar{Y}_{4}\right)<0$ and, as a consequence, $Y_{4}$ is full rank. Then, identifying (4) as iv) in Lemma $1, \mathscr{B}=\bar{Y}$ and

$$
\mathscr{B}_{\perp}=\left[\begin{array}{ccc}
I & 0 & 0 \\
0 & I & 0 \\
0 & 0 & I \\
-\bar{Y}_{4}^{-1} \bar{Y}_{1} & -\bar{Y}_{4}^{-1} \bar{Y}_{2} & -\bar{Y}_{4}^{-1} \bar{Y}_{3}
\end{array}\right]
$$

is a basis for the null space of $\bar{Y}$, that is, $\bar{Y} \mathscr{B}_{\perp}=0$. Using Lemma 1 , (4) is equivalent to $\mathscr{B}_{\perp}^{T} \mathscr{Q} \mathscr{B}_{\perp}<0$, that can be rewritten as

$$
\left[\begin{array}{ccc}
-P & C_{z}^{T} \Omega T & 0 \\
\star & -2 T & 0 \\
\star & \star & P
\end{array}\right]+\mathrm{He}\left(\bar{Y}_{4}^{-1}\left[\begin{array}{c}
\bar{Y}_{1} \\
\bar{Y}_{2} \\
\bar{Y}_{3}
\end{array}\right]\left[\begin{array}{lll}
A_{c l} & B_{c l} & -I
\end{array}\right]\right)<0 .
$$

Last inequality is, again, in the form $i v$ ) of Lemma 1 and, computing a basis for the null space of $\left[\begin{array}{lll}A_{c} & B_{c l} & -I\end{array}\right]$, the following equivalent condition can be obtained

$$
\left[\begin{array}{cc}
I & 0 \\
0 & I \\
A_{c l} & B_{c l}
\end{array}\right]^{T}\left[\begin{array}{ccc}
-P & C_{z}^{T} \Omega T & 0 \\
\star & -2 T & 0 \\
\star & \star & P
\end{array}\right]\left[\begin{array}{cc}
I & 0 \\
0 & I \\
A_{c l} & B_{c l}
\end{array}\right]<0
$$

and can be rewritten as

$$
\left[\begin{array}{cc}
A_{c l}^{T} P A_{c l}-P & A_{c l}^{T} P B_{c l}+C_{z}^{T} \Omega T \\
\star & B_{c l}^{T} P B_{c l}-2 T
\end{array}\right]<0 .
$$

Multiplying (6) on left by $\left[x^{T} \phi(z)^{T}\right]$ and on the right by the transpose, one has

$$
\begin{gathered}
x^{T} A_{c l}^{T} P A_{c l} x-x^{T} P x+\phi(z)^{T} T \Omega C_{z} x+\phi(z)^{T} B_{c l}^{T} P A_{c l} x \\
+x^{T} A_{c l}^{T} P B_{c l} \phi(z)+x^{T} C_{z}^{T} \Omega T \phi(z)+\phi(z)^{T} B_{c l}^{T} P B_{c l} \phi(z) \\
-2 \phi(z)^{T} T \phi(z)<0
\end{gathered}
$$

which corresponds to

$$
V(x(k+1))-V(x(k))-2 \phi(z(k))^{T} T[\phi(z(k))-\Omega z(k)]<0,
$$

when $V(x)=x^{T} P x$ is used as a quadratic Lyapunov function for system (1). Under Assumption 1, the last term on the left-hand side of the above inequality is always positive for any diagonal $T>0$, assuring that there exist positive $\alpha_{1}$ and $\alpha_{2}$ such that

$$
\alpha_{1}\|x\|<V(x)<\alpha_{2}\|x\|
$$

and $\Delta V$ is strictly negative along the trajectories of (1)-(3). Therefore, the system (1) in closed-loop is asymptotically globally stable.

The inequality in (4) is an LMI because $\bar{Y}_{i}, i=1, \ldots, 4$, are fixed matrices. As a consequence, it is expected that the results tend to be conservative since no general rule is known to choose suitable matrices $\bar{Y}_{i}, i=1, \ldots, 4$. Following the lines in [20], one alternative to solve the problem is the introduction of relaxations, as presented in the next theorem.

Theorem 2 Let $\bar{Y}=\left[\begin{array}{llll}0 & 0 & - & I\end{array}\right]$ and

$$
A_{c l}=\frac{1}{\rho} A+B_{u} \hat{K} C_{y}, \quad B_{c l}=\frac{1}{\rho} B_{\phi}+B_{u} \hat{L},
$$


where $\hat{K}=K / \rho, \hat{L}=L / \rho$ and $\rho$ is a positive scalar. Then, the inequalities of Theorem 1 always have a feasible solution with a finite value for $\rho$.

Proof: Consider the above expression for $\mathrm{Acl}, \mathrm{Bcl}$, and adopt the proposed choice of $\bar{Y}$, fixing $Y_{3}=-Y_{4}=I / 2$ and $K=L=0$ in inequality (4), one obtains

$$
\left[\begin{array}{cccc}
-P & C_{z}^{T} \Omega T & 0 & A^{T} / \rho \\
\star & -2 T & 0 & B_{\phi}^{T} / \rho \\
\star & \star & P-I & 0 \\
\star & \star & \star & -I
\end{array}\right]<0,
$$

or, equivalently (with a Schur complement),

$$
\left[\begin{array}{ccc}
P-\left(A^{T} A\right) / \rho^{2} & -C_{z}^{T} \Omega T-\left(A^{T} B_{\phi}\right) / \rho^{2} & 0 \\
\star & 2 T-\left(B_{\phi}^{T} B_{\phi}\right) / \rho^{2} & 0 \\
\star & \star & I-P
\end{array}\right]>0 .
$$

For a sufficiently large $\rho$, the feasibility of the above inequality can be assured by choosing $P=\varepsilon I, \varepsilon \in(0,1)$, such that

$$
\left[\begin{array}{ccc}
\varepsilon I & -C_{z}^{T} \Omega T & 0 \\
\star & 2 T & 0 \\
\star & \star & (1-\varepsilon) I
\end{array}\right]>0
$$

The positivity of block $(3,3)$ is assured for $\varepsilon \in(0,1)$, while for the upper diagonal block, using Schur complement, one must choose $T=\eta I$, with $\eta$ small enough such that $\varepsilon I>$ $\eta C_{z}^{T} \Omega \Omega C_{z}$.

From the above result, $\rho$ can be viewed as a relaxation parameter assuring that, with the choices given in Theorem 2 , Theorem 1 always provides a feasible solution (associated with a sufficiently large $\rho$ ). Note, however, that the closedloop stability requires the conditions to hold with $\rho=1$ (i.e., when the modified closed-loop matrices given in (8) become equal to the ones of the original system). In view of that, next theorem provides an important result for the implementation of an iterative procedure based on the conditions of Theorem 1.

Theorem 3 If the conditions of Theorem 1 with $A_{c l}$ and $B_{c l}$ given in (8) hold for some $\rho>0$, then they are also hold for any $\bar{\rho}>\rho$ if

$$
\operatorname{He}\left(\left[\begin{array}{l}
Y_{1} \\
Y_{2}
\end{array}\right]\left[\begin{array}{ll}
\bar{Y}_{1} & \bar{Y}_{2}
\end{array}\right]\right) \geq 0 .
$$

Proof: Consider that the conditions of Theorem 1 are feasible for a given $\rho$. Therefore, condition (6), with $A_{c l}$ and $B_{c l}$ given in (8), holds. Multiplying on both sides by $\operatorname{diag}(\rho I, \rho I)$, yields

$$
\left[\begin{array}{cc}
A_{c l}^{T} P A_{c l}-\rho^{2} P & A_{c l}^{T} P B_{c l}+\rho^{2} C_{z}^{T} \Omega T \\
\star & B_{c l}^{T} P B_{c l}-2 \rho^{2} T
\end{array}\right]<0,
$$

which can be rewritten as

$$
-\left[\begin{array}{cc}
A_{c l}^{T} P A_{c l} & A_{c l}^{T} P B_{c l} \\
\star & B_{c l}^{T} P B_{c l}
\end{array}\right]+\rho^{2} \underbrace{\left[\begin{array}{cc}
P & -C_{z}^{T} \Omega T \\
\star & 2 T
\end{array}\right]}_{\Psi}>0 .
$$

Clearly, the above condition remains feasible for any $\bar{\rho}>\rho$ if $\Psi$ is positive semi-definite. Note that a necessary condition for the feasibility of (4) is (first two blocks of rows and columns)

$$
\left[\begin{array}{cc}
-P & C_{z}^{T} \Omega T \\
\star & -2 T
\end{array}\right]+\operatorname{He}\left(\left[\begin{array}{l}
Y_{1} \\
Y_{2}
\end{array}\right]\left[\begin{array}{ll}
\bar{Y}_{1} & \bar{Y}_{2}
\end{array}\right]\right)<0
$$

which guarantees that $\Psi \geq 0$ if (9) is feasible, concluding the proof.

Using the results of Theorem 3, and the relaxations given in (8), one can guarantee that, if Theorem 1 , solved jointly with (9), provides a feasible solution with $\rho \leq 1$, the stability of the closed-loop system is assured. Another possibility that guarantees (9) without any additional LMI is simply to enforce $Y_{1}=Y_{2}=0$ in the conditions of Theorem 1. This choice is performed in the examples of Section IV.

The main contribution of this paper, constructed in terms of the theoretical results presented in Theorems 1, 2 and 3, is Algorithm 1, which is an iterative procedure for the stabilization of system (1) by means of the feedback control law (3). As input parameters, one has $i t_{\max }$, the maximum number of iterations, and $\bar{Y}=Y_{0}$, the initial condition. The results of Theorem 2 and 3 guarantee that the procedure provides a feasible solution with a finite value for $\rho$. Moreover, the convergence in terms of $\rho$ is monotonically non increasing, as proved in the next theorem.

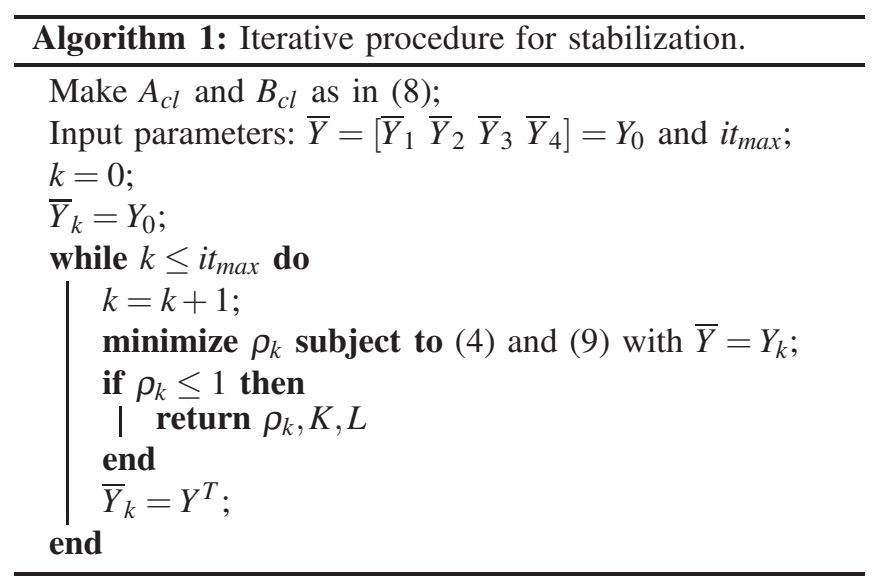

Theorem 4 The sequence of values $\rho_{k}$ provided by Algorithm 1 is non-increasing.

Proof: Suppose that at iteration $k$ a finite value for $\rho_{k}$, associated to the variables $P_{k}, T_{k}$ and $Y_{k}$, has been obtained. Since $\operatorname{He}(Y \bar{Y})=\operatorname{He}\left(\bar{Y}^{T} Y^{T}\right)$, the choice $\bar{Y}_{k+1}=Y_{k}$ assures that $\rho_{k+1}$ cannot increase, that is, $\rho_{k+1} \leq \rho_{k}$.

Note that the convergence of Algorithm 1 is only local, depending on the initial choice $\bar{Y}=Y_{0}=\left[\begin{array}{llll}\bar{Y}_{1} & \bar{Y}_{2} & \bar{Y}_{3} & \bar{Y}_{4}\end{array}\right]$.

\section{NUMERICAL EXAMPLES}

In this section, numerical examples illustrate the performance of the proposed conditions when compared with methods available in the literature. The LMI conditions were programmed using MATLAB, Yalmip [21] and the solver Mosek [22] in a PC running Windows 10 core 17. 
Example 1: This example, borrowed from [15], is obtained from the discretization (sampling time $T=0.01 s$ ) of a continuous-time model, resulting on

$$
\begin{gathered}
A=v\left[\begin{array}{ll}
0.8000 & 0.0500 \\
0.1000 & 1.0500
\end{array}\right], \quad B_{\phi}=\left[\begin{array}{l}
0.0096 \\
0.0096
\end{array}\right], \\
B_{u}=\left[\begin{array}{l}
0.0300 \\
0.0300
\end{array}\right], \quad C_{z}=\left[\begin{array}{ll}
0.8 & 0.8
\end{array}\right]
\end{gathered}
$$

where $v$ is an additional positive scalar parameter. The objective is to determine the maximum value of $v$ such that the system can be stabilized by state feedback (i.e., $C_{y}=I$ ) considering different values of $\Omega$. Algorithm 1 (denoted by A1), with $\bar{Y}_{0}=\left[\begin{array}{llll}0 & 0 & -I I\end{array}\right]$ and $i t_{\max }=8$, is compared with the conditions in [15] (denoted by LJD15) and the results are presented in Figure 1. The number of LMI rows $R$ used by Al1 and LJD15 is 10 and 15, respectively, and the number of variables $V$ is 20 and 18 .

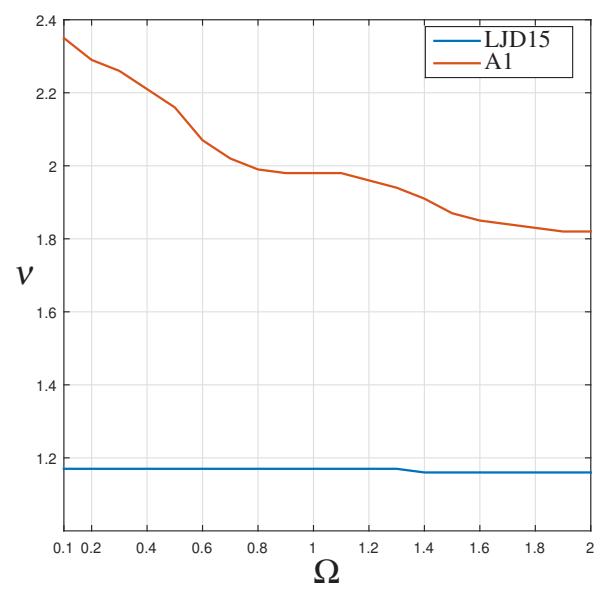

Fig. 1. Maximum values of $v$ versus $\Omega$ for state feedback stabilization using A1 (8 iterations, red line) and LJD15 (blue line) in Example 1.

Note that Algorithm 1 provides larger stabilization regions in terms of $v$ for the whole range of $\Omega$ considered with $v$ tending to smaller values as $\Omega$ increases.

Example 2: In this example the proposed synthesis conditions are evaluated and compared with methods from the literature in terms of a statistical analysis in the sectorbounded case. To accomplish this task, a database of systems as in (1) with matrices randomly generated was created as follows

i) Matrices $A, B_{\phi}$ and $C_{z}$ are created (using a gaussian distribution) such that system (1) fulfills the stability condition [2, Theorem 2];

ii) Matrices $B_{u}, C_{y}$ and $K$ are generated such that $A-$ $B_{u} K C_{y}$ is not Schur stable;

iii) Matrices $A \leftarrow A-B_{\phi} K C_{y}, B_{\phi}, C_{y}, C_{z}$ and $B_{u}$ are included in the database.

Considering $n_{x} \in\{2,5\}, n_{u}=n_{z}=n_{y}=1$ and $\Omega \in$ $\{0.5,1,2.1445,11.4301\}$ (representing 26.56, 45, 65 and 85 degrees), 100 systems were generated for each case (total of
1600 systems). The important feature of the database is that all systems are guaranteed to be stabilized by the control law (3) with $L=0$. The objective is compare Algorithm 1 with $\bar{Y}_{0}=\left[\begin{array}{lll}0 & 0 & -I I\end{array}\right], i t_{\max }=15$ and $L$ as variable or fixed as zero $(L=0)$, with [13, Theorem 1] (denoted by KB17) considering the output feedback control law. Note that KB17 is not able to design the gain $L$.

Table I shows the average percentage of stabilized systems for each value of $\Omega$, the associated numerical complexity given in terms of mean time ( $t$, in milliseconds) required to test each system and the average number of iterations $\left(i t_{a}\right)$ demanded by A1. Although the proposed method is more time consuming, the results in terms of stabilized systems are clearly superior, specially for higher values of $\Omega$.

TABLE I

AVERAGE PERCENTAGE OF STABILIZED SYSTEMS BY A $1(L=0$ OR

$L \neq 0$ ) AND KB 17 , THE MEAN TIME ( $t$, IN MILLISECONDS) TO TEST EACH SYSTEM, AND THE AVERAGE NUMBER OF ITERATIONS DEMANDED BY A 1 .

\begin{tabular}{c|cc|ccc|ccc}
\hline & \multicolumn{2}{|c|}{ KB17 } & \multicolumn{3}{c|}{$\mathrm{A}_{L \neq 0}$} & \multicolumn{3}{c}{$\mathrm{A} 1_{L=0}$} \\
\hline$\Omega$ & $\%$ & $t$ & $\%$ & $i t_{a}$ & $t$ & $\%$ & $i t_{a}$ & $t$ \\
\hline 0.5 & 79.75 & 2.7 & 99.50 & 1.2 & 5.1 & 99.75 & 1.2 & 5.2 \\
1 & 67 & 2.4 & 99.75 & 1.3 & 5.4 & 99.75 & 1.4 & 5.7 \\
2.14 & 58.5 & 2.6 & 99.25 & 1.4 & 6.4 & 99.75 & 1.5 & 6.2 \\
11.43 & 46 & 3.0 & 99.25 & 2.4 & 13.4 & 99.75 & 2.5 & 12.9 \\
\hline
\end{tabular}

A more detailed analysis regarding the number of iterations performed by A1 is presented in Table II, where several values of $i t_{\max }$ were considered. Values in bold indicate that the maximum number of systems that can be stabilized with $i t_{\max }=15$ has already been detected. Note that only the case $\Omega=11.56$ requires more than 8 iterations to achieve the maximum number of systems stabilized with $i t_{\max }=15$. Another interesting property revealed by Table II is that A1 is capable to outperform KB17 with a single iteration, that is, without resorting to the iterative procedure, in the cases $\Omega \in\{0.5,1,2.14\}$. Finally, 4 iterations are enough to stabilize around $95 \%$ of the database.

With respect to consider or not the gain $L$ in A1, note that, except for the case $\Omega=1, A 1$ with $L=0$ provided slightly

\section{TABLE II}

AVERAGE PERCENTAGE OF STABILIZED SYSTEMS BY A $1(L=0$ OR $L \neq 0$ ) CONSIDERING SEVERAL VALUES OF $i t_{\text {max }}$. VALUES IN BOLD INDICATE THAT THE MAXIMUM NUMBER OF SYSTEMS THAT CAN BE

\begin{tabular}{|c|c|c|c|c|c|c|c|c|}
\hline \multirow[b]{3}{*}{$i t_{\max }$} & \multirow{2}{*}{\multicolumn{4}{|c|}{$\begin{array}{c}\mathrm{A} 1_{L \neq 0} \\
\Omega\end{array}$}} & \multicolumn{4}{|c|}{$\mathrm{A} 1_{L=0}$} \\
\hline & & & & & & & & \\
\hline & 0.5 & 1.0 & 2.14 & 11.43 & 0.5 & 1.0 & 2.14 & 11.43 \\
\hline 1 & 83.00 & 70.25 & 62.00 & 34.50 & 78.75 & 66.50 & 56.00 & 29.00 \\
\hline 2 & 97.25 & 98.00 & 96.00 & 56.75 & 97.00 & 95.00 & 93.75 & 50.25 \\
\hline 3 & 99.00 & 99.25 & 98.25 & 84.75 & 99.00 & 98.25 & 99.00 & 79.25 \\
\hline 4 & 99.25 & 99.50 & 98.75 & 94.75 & 99.50 & 99.00 & 99.25 & 95.50 \\
\hline 5 & 99.25 & 99.50 & 99.00 & 97.25 & 99.75 & 99.00 & 99.50 & 98.00 \\
\hline 6 & 99.50 & 99.75 & 99.00 & 97.75 & 99.75 & 99.50 & 99.50 & 99.00 \\
\hline 7 & 99.50 & 99.75 & 99.00 & 98.00 & 99.75 & 99.75 & 99.75 & 99.50 \\
\hline 8 & 99.50 & 99.75 & 99.25 & 98.75 & 99.75 & 99.75 & 99.75 & 99.50 \\
\hline 11 & 99.50 & 99.75 & 99.25 & 99.00 & 99.75 & 99.75 & 99.75 & 99.75 \\
\hline 14 & 99.50 & 99.75 & 99.25 & 99.25 & 99.75 & 99.75 & 99.75 & 99.75 \\
\hline
\end{tabular}
STABILIZED WITH $i t_{\text {max }}=15$ HAS ALREADY BEEN DETECTED. 
better results, which seems to be counter intuitive. The reason for this fact is that A1 tested with $L$ may not achieve the same value of $\rho$ when tested with $L=0$, except in the first iteration. This fact is corroborated by the row $i t_{\max }=1$ in Table II. Actually, as the iterative procedure evolves, different values of $\rho$ can be obtained, since the problems may have different local minimums.

Example 3: In this example the problem of state-feedback $\left(C_{y}=I\right)$ is investigated using the same database of systems from the previous example. Clearly, $K C_{y}$ is a valid statefeedback gain and, as a consequence, all systems of the database can also be stabilized by state-feedback. Regarding the conditions to be tested, the method from [15, Theorem 3.1] (denoted by LJD15) is included in the comparisons. The results of A1 (using the same settings employed in Example 2), KB17 ([13, Lemma 4]) and LDJ15 are presented in Table III.

Regarding the results, it is important to mention that KB17 is not able to feedback the nonlinearity, and this fact is advantageous when producing synthesis conditions. As a matter of fact, the feedback of $\phi(z)$ poses technical difficulties to express the design conditions in terms of LMIs, in general circumvented with the introduction of conservativeness. This explains the superior performance of KB17 with respect to LDJ15. On the other hand, the approach proposed in this paper is completely different, and the same methodology can be used to investigate state- and output-feedback control design. Again one can observe counter intuitive results of A1 in some cases, that is, output-feedback outperforming statefeedback. As explained before, except at the first iteration, the evolution of $\rho$ can be completely different in both cases. For instance, considering $i t_{\max }=1$, the following results are obtained respectively for $\Omega \in\{0.5,1,2.14,11.43\}$ : $\{85.50 \%$, $71.50 \%, 63.00 \%, 34.75 \%\}(L \neq 0)$ and $\{86.00 \%, 71.50 \%$, $62.25 \%, 33.00 \%\}(L=0)$, which are equal or better than output-feedback (see row $i t_{\max }=1$ of Table II).

Nevertheless, ultimately, state-feedback gains could be produced for this experiment simply considering $K C_{y}$ with $K$ obtained in Example 2.

Example 4: As last experiment, the problem of decentralized state-feedback $\left(C_{y}=I\right)$ is investigated. Only the systems with $n_{x}=5$ of the database are tested and the following masks for the gain $K$ are considered:

$$
\begin{aligned}
& K=\left[\begin{array}{lllll}
1 & 1 & 1 & 1 & 1
\end{array}\right] \quad\left(M_{1}\right) \\
& K=\left[\begin{array}{lllll}
1 & 1 & 0 & 1 & 1
\end{array}\right] \quad\left(M_{2}\right) \\
& K=\left[\begin{array}{lllll}
1 & 0 & 1 & 0 & 1
\end{array}\right] \quad\left(M_{3}\right) \\
& K=\left[\begin{array}{lllll}
1 & 0 & 0 & 0 & 1
\end{array}\right] \quad\left(M_{4}\right) \\
& K=\left[\begin{array}{lllll}
1 & 0 & 0 & 0 & 0
\end{array}\right] \quad\left(M_{5}\right) \\
& K=\left[\begin{array}{lllll}
0 & 0 & 0 & 0 & 1
\end{array}\right] \quad\left(M_{6}\right)
\end{aligned}
$$

where 1 indicates that the corresponding entry is to be designed. To guarantee that all systems can be stabilized by the proposed masks, the following adjustment is performed in the dynamic matrices of the systems in the database:

$$
A_{d c}=A+B_{u}\left(K C_{y}-K C_{y} \circ M_{i}\right)
$$

where $M_{i}$ is the desired mask, $K C_{y}$ is a stabilizing statefeedback gain ( $K$ is available in the database) and $\circ$ is the Hadamard product defined as $(X \circ Y)_{i, j}=X_{i, j} Y_{i, j}$. With this change, $K_{d c}=K C_{y} \circ M_{i}$ is a decentralized stabilizing gain according to mask $M_{i}$ because

$$
\begin{aligned}
A_{d c}+B_{u}\left(K C_{y} \circ M_{i}\right) & =A+B_{u}\left(K C_{y}-K C_{y} \circ M_{i}\right)+B_{u}\left(K C_{y} \circ M_{i}\right) \\
& =A+B_{u} K C_{y}
\end{aligned}
$$

The condition in KB17 is adapted to cope with decentralized using constraints in the optimization variables, following the strategy proposed in [23]. A1 and LJD15 are more easily adapted since the control gain is an optimization variable of the problem. The results show that KB17 is not suitable for the design of decentralized gains, specially when the number of null entries is large. This can be explained by the fact that the Lyapunov matrix needs to be constrained, being diagonal in some cases (masks $M_{5}$ and $M_{6}$ ). The condition from LDJ15 presents some loose of performance when compared to full state-feedback (mask $M_{1}$ ), while A1 keeps the same level of accuracy, showing even less conservative results when dealing with decentralized control.

\section{CONCLUSIONS}

This work proposed sufficient LMI conditions based on a quadratic Lyapunov function in conjunction with an iterative approach to stabilize discrete-time Lur'e systems with sectorbounded nonlinearities. Output or state feedback control laws can be designed, taking into account one gain proportional to the linear output and another gain that feedbacks the nonlinearity. The iterative algorithm, with local convergence, explores a relaxation variable $\rho$ and the fact that the Lyapunov and the closed-loop matrices appear affinely in the conditions. Therefore, the control gains are treated directly as optimization variables of the problem, what can be a great advantage when designing structured controllers. Examples showed a good trade-off between accuracy and computational effort in comparison with other methods from the literature when dealing with state or output-feedback, and decentralized control.

\section{REFERENCES}

[1] H. K. Khalil, Nonlinear Systems, 3rd ed. Upper Saddle River, NJ: Prentice Hall, 2002.

[2] C. A. C. Gonzaga, M. Jungers, and J. Daafouz, "Stability analysis of discrete-time Lur'e systems," Automatica, vol. 48, no. 9, pp. 22772283, Sep. 2012.

[3] N. S. Ahmad, W. P. Heath, and G. Li, "LMI-based stability criteria for discrete-time Lur'e systems with monotonic, sector- and sloperestricted nonlinearities," IEEE Transactions on Automatic Control, vol. 58, no. 2, pp. 459-465, Feb. 2013.

[4] P. Park, "A revisited Popov criterion for nonlinear Lur'e systems with sector-restrictions," International Journal of Control, vol. 68, no. 3, pp. 461-469, 1997.

[5] J. Park, S. Y. Lee, and P. Park, "A less conservative stability criterion for discrete-time Lur'e systems with sector and slope restrictions," IEEE Transactions on Automatic Control, vol. 64, no. 10, pp. 43914395, Oct. 2019.

[6] V. Kapila and W. M. Haddad, "A multivariable extension of the Tsypkin criterion using a Lyapunov-function approach," IEEE Transactions on Automatic Control, vol. 41, no. 1, pp. 149-152, jan 1996. 
TABLE III

AVERAGE PERCENTAGE OF STABILIZED SYSTEMS By A $1(L=0$ or $L \neq 0)$, LDJ15 $(L=0$ OR $L \neq 0)$ AND KB17, THE MEAN TIME $(t$, IN MILLISECONDS) TO TEST EACH SYSTEM, AND THE AVERAGE NUMBER OF ITERATIONS DEMANDED BY A1.

\begin{tabular}{|c|c|c|c|c|c|c|c|c|c|c|c|c|}
\hline \multirow[b]{2}{*}{$\Omega$} & \multicolumn{2}{|c|}{ KB17 } & \multicolumn{3}{|c|}{$\mathrm{A} 1_{L \neq 0}$} & \multicolumn{3}{|c|}{$\mathrm{A} 1_{L=0}$} & \multicolumn{2}{|c|}{ LJD15 $_{L \neq 0}$} & \multicolumn{2}{|c|}{$\operatorname{LJD}_{15} 5_{L=0}$} \\
\hline & $\%$ & $\bar{t}$ & $\%$ & $i t_{\text {ave }}$ & $\mathrm{t}$ & $\%$ & $i t_{\text {ave }}$ & $\mathrm{t}$ & $\%$ & $\mathrm{t}$ & $\%$ & $\mathrm{t}$ \\
\hline 0.5 & 98 & 3.4 & 99.25 & 1.16 & 7.9 & 100 & 1.16 & 5.2 & 90.5 & 5.6 & 89.5 & 3.8 \\
\hline 1 & 94.5 & 3.1 & 99 & 1.32 & 7.9 & 99.25 & 1.31 & 5.3 & 83 & 5.9 & 82.25 & 3.7 \\
\hline 2.14 & 91.5 & 3.3 & 99 & 1.42 & 8.5 & 99.75 & 1.43 & 6.2 & 83.25 & 5.2 & 79.75 & 3.9 \\
\hline 11.43 & 94 & 2.7 & 99 & 2.41 & 14.2 & 100 & 2.30 & 13.5 & 92 & 3.6 & 88.75 & 3.6 \\
\hline
\end{tabular}

TABLE IV

NUMBER OF STABILIZED SYSTEMS WITH $n_{x}=5$ BY A1 $(L=0$ OR $L \neq 0)$, LDJ $15(L=0$ OR $L \neq 0)$ AND KB 17 IN THE PROBLEM OF DECENTRALIZED STATE-FEEDBACK USING THE MASKS GIVEN IN (10).

\begin{tabular}{c|c|c|c|c|c|c}
\hline$\Omega$ & Mask & $\mathrm{KB} 17$ & $\mathrm{~A}_{L \neq 0}$ & $\mathrm{~A}_{L=0}$ & $\mathrm{LJD} 15_{L \neq 0}$ & $\mathrm{LJD} 15_{L=0}$ \\
\hline \multirow{5}{*}{0.5} & $M_{1}$ & 98 & 99 & 99 & 85 & 84 \\
& $M_{2}$ & 28 & 99 & 99 & 84 & 82 \\
& $M_{3}$ & 8 & 100 & 100 & 85 & 82 \\
& $M_{4}$ & 1 & 100 & 100 & 84 & 78 \\
& $M_{5}$ & 2 & 100 & 100 & 82 & 76 \\
& $M_{6}$ & 0 & 100 & 100 & 82 & 75 \\
\hline \multirow{6}{*}{1} & $M_{1}$ & 94 & 99 & 99 & 75 & 73 \\
& $M_{2}$ & 31 & 100 & 100 & 74 & 70 \\
& $M_{3}$ & 13 & 99 & 99 & 71 & 66 \\
& $M_{4}$ & 6 & 100 & 100 & 66 & 64 \\
& $M_{5}$ & 4 & 100 & 100 & 66 & 62 \\
& $M_{6}$ & 3 & 100 & 100 & 66 & 63 \\
\hline \multirow{6}{*}{2.14} & $M_{1}$ & 94 & 98 & 98 & 76 & 73 \\
& $M_{2}$ & 20 & 99 & 99 & 73 & 67 \\
& $M_{3}$ & 3 & 100 & 100 & 70 & 65 \\
& $M_{4}$ & 3 & 100 & 100 & 65 & 56 \\
& $M_{5}$ & 4 & 100 & 100 & 60 & 49 \\
& $M_{6}$ & 2 & 100 & 100 & 60 & 46 \\
\hline \multirow{6}{*}{11.52} & $M_{1}$ & 96 & 98 & 98 & 97 & 93 \\
& $M_{2}$ & 12 & 98 & 98 & 87 & 80 \\
& $M_{3}$ & 4 & 98 & 98 & 80 & 69 \\
& $M_{4}$ & 3 & 98 & 98 & 74 & 59 \\
& $M_{5}$ & 0 & 99 & 99 & 70 & 55 \\
& $M_{6}$ & 4 & 99 & 99 & 71 & 55 \\
\hline \multirow{6}{*}{}
\end{tabular}

[7] V. F. Montagner, R. C. L. F. Oliveira, T. R. Calliero, R. A. Borges, P. L. D. Peres, and C. Prieur, "Robust absolute stability and stabilization based on homogeneous polynomially parameter-dependent Lur'e functions," in Proceedings of the 2007 American Control Conference, New York, NY, USA, Jul. 2007, pp. 6021-6026.

[8] C. Yang, Q. Zhang, and L. Zhou, "Lur'e Lyapunov functions and absolute stability criteria for Lur'e systems with multiple nonlinearities," International Journal of Robust and Nonlinear Control, vol. 17, no. 9, pp. 829-841, Jun. 2007.

[9] R. E. Kalman, "Lyapunov functions for problem of Lur'e in automatic control," Proceedings of the National Academy of Sciences of the United States of America, vol. 49, no. 2, pp. 201-205, Feb. 1963.

[10] N. S. Ahmad and J. Carrasco, "Convex LMI approach for stability of critically stable systems with slope-restricted nonlinearities," Proceedings of the 55th IEEE Conference on Decision and Control, pp. 3228-3233, Dec. 2016.

[11] J. Carrasco, M. C. Turner, and W. P. Heath, "Zames-Falb multipliers for absolute stability: From O'Shea's contribution to convex searches," European Journal of Control, vol. 28, pp. 1-19, Mar. 2016.

[12] E. B. Castelan, S. Tarbouriech, and I. Queinnec, "Control design for a class of nonlinear continuous-time systems," Automatica, vol. 44, no. 8, pp. 2034-2039, Mar. 2008.

[13] K. K. Kim and R. D. Braatz, "Robust static and fixed-order dynamic output feedback control of discrete-time parametric uncertain Luré systems: Sequential SDP relaxation approaches," Optimal Control Applications and Methods, vol. 38, no. 1, pp. 36-58, January/February 2017

[14] — "Observer-based output feedback control of discrete-time Luré systems with sector-bounded slope-restricted nonlinearities," International Journal of Robust and Nonlinear Control, vol. 24, no. 16, pp. 2458-2472, Nov. 2014.

[15] J. Louis, M. Jungers, and J. Daafouz, "Sufficient LMI stability conditions for Lur'e type systems governed by a control law designed on their Euler approximate model," International Journal of Control, vol. 88, no. 9, pp. 1841-1850, Feb. 2015.

[16] M. Jungers and J. Daafouz, "Dynamic output absolute stabilization of a discrete-time switched Lur'e system," IFAC-PapersOnLine, vol. 51, no. 25, pp. 359-364, Sep. 2018, proceedings of the Joint 9th IFAC Symposium on Robust Control Design (ROCOND'18), and 2nd IFAC Workshop on Linear Parameter Varying Systems (LPVS'18).

[17] S. M. Lee and J. H. Park, "Robust stabilization of discrete-time nonlinear Lur'e systems with sector and slope restricted nonlinearities," Applied Mathematics and Computation, vol. 200, pp. 429-436, 2008.

[18] S. Boyd, L. El Ghaoui, E. Feron, and V. Balakrishnan, Linear Matrix Inequalities in System and Control Theory. Philadelphia, PA: SIAM Studies in Applied Mathematics, 1994.

[19] M. C. de Oliveira and R. E. Skelton, "Stability tests for constrained linear systems," in Perspectives in Robust Control, ser. Lecture Notes in Control and Information Science, S. O. Reza Moheimani, Ed. New York, NY: Springer-Verlag, 2001, vol. 268, pp. 241-257.

[20] A. Felipe, R. C. L. F. Oliveira, and P. L. D. Peres, "An iterative LMI based procedure for robust stabilization of continuous-time polytopic systems," in Proceedings of the 2016 American Control Conference, Boston, MA, USA, Jul. 2016, pp. 3826-3831.

[21] J. Löfberg, "YALMIP: A toolbox for modeling and optimization in MATLAB," in Proceedings of the 2004 IEEE International Symposium on Computer Aided Control Systems Design, Taipei, Taiwan, Sep. 2004, pp. 284-289, http://yalmip.github.io.

[22] E. D. Andersen and K. D. Andersen, "The MOSEK interior point optimizer for linear programming: An implementation of the homogeneous algorithm," in High Performance Optimization, ser. Applied Optimization, H. Frenk, K. Roos, T. Terlaky, and S. Zhang, Eds. Springer US, 2000, vol. 33, pp. 197-232, http://www.mosek.com.

[23] J. C. Geromel, J. Bernussou, and P. L. D. Peres, "Decentralized control through parameter space optimization," Automatica, vol. 30, no. 10, pp. 1565-1578, Oct. 1994. 